Document downloaded from:

http://hdl.handle.net/10251/157214

This paper must be cited as:

Torres, V.; Ortuño, R.; Rodríguez-Ulibarri, P.; Griol Barres, A.; Martínez Abietar, AJ.; Navarro-Cía, M.; Beruete, M.... (2014). Mid-infrared Plasmonic Inductors. OSA. 1-2. https://doi.org/10.1364/CLEO_QELS.2014.FTu1C.3

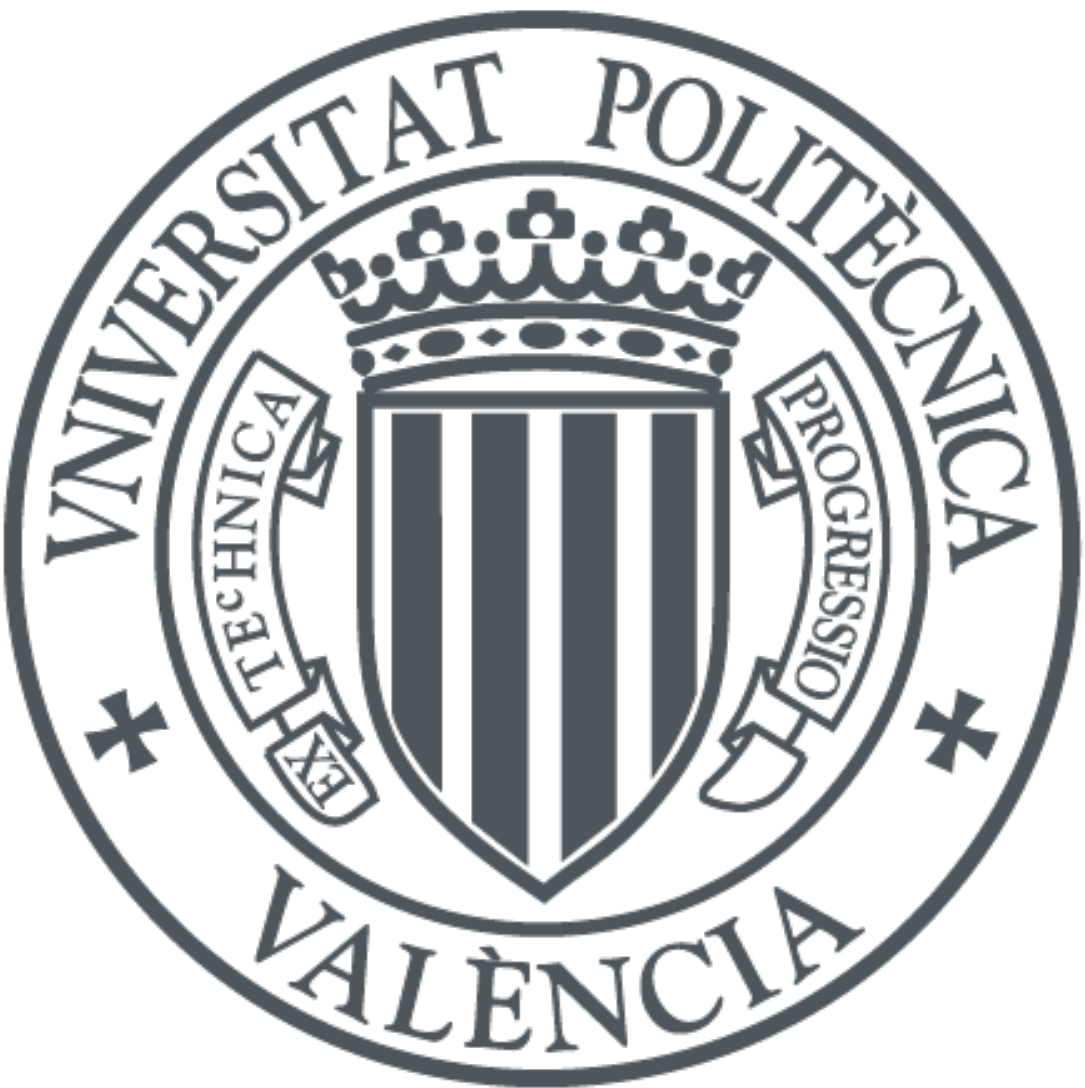

The final publication is available at

https://doi.org/10.1364/CLEO_QELS.2014.FTu1C.3

Copyright OSA

Additional Information 


\title{
Mid-infrared Plasmonic Inductors
}

\author{
Víctor Torres' ${ }^{1}$, Rubén Ortuño ${ }^{1}$, Pablo Rodríguez-Ulibarri' ${ }^{1}$, Amadeu Griol ${ }^{2}$, Alejandro Martínez², Miguel \\ Navarro-Cía ${ }^{3}$, Miguel Beruete ${ }^{1}$, and Mario Sorolla ${ }^{1}$ \\ ${ }^{1}$ TERALAB(MmW- THz - IR\&Plasmonics Laboratory), Universidad Pública de Navarra, 31006, Pamplona, Spain, ${ }^{2}$ Nanophotonics \\ Technology Center, Universitat Politècnica de València, ${ }^{3}$ Optical and Semiconductor Devices Group, \\ Department of Electrical and Electronic Engineering, Imperial College London \\ Author e-mail address: victor.torres@unavarra.es
}

\begin{abstract}
Expanding ideas from microwaves, we demonstrate experimentally a terahertz inductor by using meander-lines in a canonical extraordinary transmission (ET) hole array leading to a strong resonance's redshift and an unprecedented enlargement of the operation bandwidth. OCIS codes: (160.3918) Metamaterials; (250.5403) Plasmonics; (260.2110) Electromagnetic optics
\end{abstract}

\section{Introduction}

In the last years, we have witnessed a flurry of activity in the field of plasmonics due to their vast potential applications [1]. Plasmonic phenomena were initially explained in terms of coupled resonant states. However, plasmonic nanocircuits are nowadays also explained in terms of circuit concepts such as lumped elements, characteristic impedance and transmission line theory [2,3] giving rise to the concept of metatronics [4-6].

Here, considering the fact that an inductance exists wherever a current loop resides, we demonstrate experimentally a terahertz inductor in frequencies as high as $17 \mathrm{THz}$ by using meander-lines in a canonical ET hole array. Furthermore, we model the response of ET hole arrays loaded with inductors of different turns from a metatronics perspective, corroborating the experimental results. The proposed structure provokes a strong redshift of the resonant peak and an unprecedented enlargement of the operation bandwidth even for a 1-turn inductor.

The proposed inductance could be used as a building block for sophisticated devices in the THz and midinfrared regimes, finding potential application in imaging or sensing applications.

\section{Results}

SEM images of Fig. 1a depict the three samples fabricated over a Si wafer with 3-, 1- and 0-turn meander lines. All structures were milled with the same hole and meander's width dimensions shown in the sketched unit cell along with the rest of the structural dimensions and the different material layers that form the samples.

Experimental measurements were performed in the vacuum chamber of a FTIR equipped with an optical bench and a He-cooled bolometer detector. An impinging vertical linear polarization allows the meanders to get excited by the induced current running along them. Experimental results normalized with respect to the transmission through a

bare silicon wafer can be seen in Fig. $1 \mathrm{~b}$ for all prototypes. The resonance frequency corresponds to that at which the impinging light couples to a symmetric leaky mode thanks to the additional momentum provided by the lattice [7]. This peak of transmission undergoes a significant redshift together with a broadening of the operation bandwidth as the number of meander turns increases. This behavior is related to the effective inductance introduced by the meanderlines [8]. Corroborative numerical results, corresponding to best fits based on estimated dimensions from SEM measurements of the samples, are also shown in Fig. 1b, with a good agreement between experiment and numerical calculations.
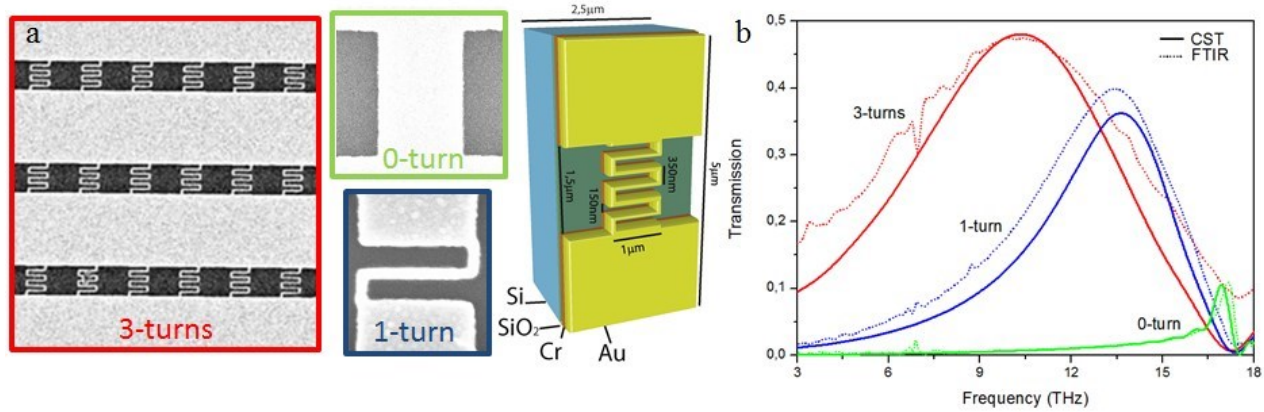

Fig. 1. a) SEM pictures of the 3-, 1- and 0-turn meandered hole arrays samples and the principal dimensions marked in the unit cell. b) Experimental and numerical results of the fabricated samples. 


\section{Discussion}

To elucidate the physical properties of the meandered hole array we have developed an equivalent circuit (EC) that models the behaviour of the fabricated samples (see Fig. 2a). Considering the virtual current loops (VCLs) formed at the apertures, the inductance $\left(\mathrm{L}_{\mathrm{ap}}\right)$ ascribed to the meanders can be rigorously extracted taking into account the inducedsurface current and the magnetic flux at each VCL [8]. The effect of adding meander-lines can be inferred from the linear trend of $\mathrm{L}_{\mathrm{ap}}$ shown at Fig.2a, where each turn adds approximately $0.45 \mathrm{pH}$. The physical meaning of the rest of parameters comprising the circuit is as follows: $Z^{(i)}$ is the background material characteristic impedance, $Z_{c}(\omega)$ models the losses in the metal and $\mathrm{C}^{(\mathrm{i})}(\omega)$ the admittance of the below-cutoff TM mode excited at each interface. Finally, the $\pi$-network of inductances $L_{p}-L_{s}-L_{p}$ accounts for the metal thickness.

The EC's accuracy is validated from the good agreement of the calculated values of the $L_{a p}$ (Fig. 2a) and the great match of the transmission between CST and EC model shown in Fig. 2b. Both results demonstrate the physical meaningful circuit representation.
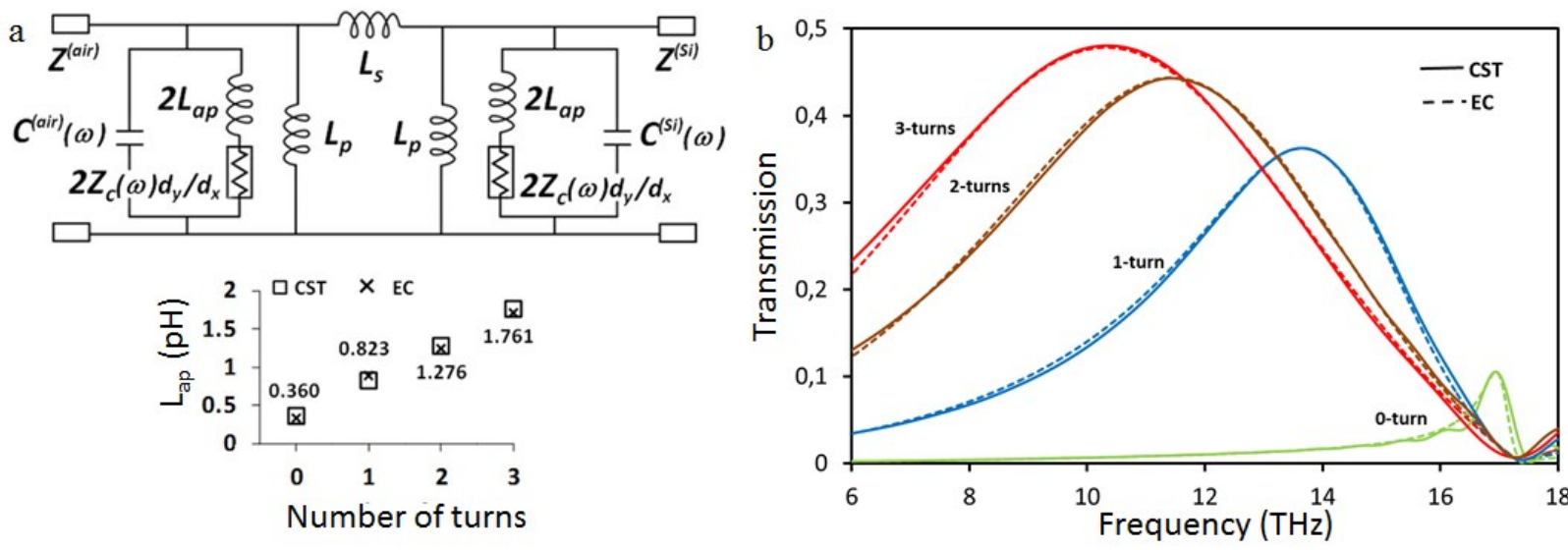

Fig. 2. a) Schematic of the EC model (top) and inductance values obtained with CST and EC model (bottom), and b) numerical results of the EC vs. CST model.

In conclusion, the importance of the result is twofold: from a fundamental viewpoint, the direct applicability of equivalent circuit concepts borrowed from microwaves is demonstrated, in frequencies as high as $17 \mathrm{THz}$ upholding unification of plasmonics and microwave concepts and allowing for a simplification of structure design and analysis; in practical terms, a broadband transmission with fractional bandwidth and efficiency as high as $97 \%$ and $48 \%$, respectively, is achieved through an area less than one hundredth the squared wavelength, which leads to an impressive accessible strong field localization that may be of great interest in sensing and imaging applications.

\section{References}

[1] R. Stanley, "Plasmonics in the mid-infrared,” Nature Photon. 6, 409-411 (2012).

[2] G. Veronis, and S. Fan, “Bends and splitters in subwavelength metal-dielectric-metal plasmonic waveguides,” Appl. Phys. Lett. 87, 131102 (2005).

[3] S. E. Kocabas, G. Veronis, D. A. B. Miller, and S. Fan, "Transmission line and equivalent circuit models for plasmonic waveguide components,” IEEE J Sel. Top. Quant. 14, 1462 (2008).

[4] N. Engheta, “Circuits with light at nanoscales: optical nanocircuits inspired by metamaterials,” Science 317, 1698-1702 (2007).

[5] Y. Sun, B. Edwards, A. Alù, and N. Engheta, "Experimental realization of optical lumped nanocircuits at infrared wavelengths,” Nature Mater. 11, 208-212 (2012).

[6] F. Monticone, N. M. Estakhri, and A. Alù, "Full control of nanoscale optical transmission with a composite metascreen,” Phys. Rev. Lett. 110, 203903 (2013).

[7] M. Beruete, M. Navarro-Cía, V. Torres, and M. Sorolla, “Redshifting extraordinary transmission by simple inductance addition,” Phys. Rev. B 84, 075140 (2011). 
[8] V. Torres, R. Ortuño, P. Rodríguez-Ulibarri, A. Griol, A. Martínez, M. Navarro-Cía, M. Beruete, and M. Sorolla, "Mid-infrared plasmonic inductors: Enhancing inductance with meandering lines,” Sci. Rep. 4, 3592 (2014). 